PROCEEDINGS OF THE

AMERICAN MATHEMATICAL SOCIETY

Volume 128, Number 3, Pages 629-636

S 0002-9939(99)05415-5

Article electronically published on October 25, 1999

\title{
FAMILIES OF IRREDUCIBLE PRINCIPALLY POLARIZED ABELIAN VARIETIES ISOMORPHIC TO A PRODUCT OF ELLIPTIC CURVES
}

\author{
VÍCTOR GONZÁLEZ-AGUILERA AND RUBí E. RODRÍGUEZ \\ (Communicated by Ron Donagi)
}

\begin{abstract}
For each $n$ greater than or equal to two, we give a family of $n$-dimensional, irreducible principally polarized abelian varieties isomorphic to a product of elliptic curves. This family corresponds to the modular curve $X_{0}(n+1)$.
\end{abstract}

\section{INTRODUCTION}

The Jacobian variety associated to a compact Riemann surface is an irreducible principally polarized abelian variety. An interesting problem, then, is to find families of Jacobians that are isomorphic to products of sub-tori. Examples of this kind of families are given in [3] and in [4].

A natural generalization is to ask for families of irreducible principally polarized abelian varieties that are isomorphic (and not only isogenous) to products of subtori.

In this short paper we prove that, for each dimension $n$, there is a family of irreducible principally polarized abelian varieties that are isomorphic to a product of $n$ elliptic curves. These families arise from the standard action of the symmetric group and are parametrized by the modular curves $X_{0}(n+1)$.

The special case of dimension three appears in [8] and in [10]. The more general case for Weyl groups will appear elsewhere.

\section{Notation}

Let $S_{n+1}$ be the symmetric group on $n+1$ letters. We let $\alpha$ and $\gamma$ denote, respectively, the permutations $\alpha=\left(\begin{array}{lllll}1 & 2 & 3 & \ldots & n+1\end{array}\right)$ and $\gamma=\left(\begin{array}{ll}1 & 2\end{array}\right)$.

Let $\mathbb{H}_{n}$ denote the Siegel upper half-space of $n \times n$ complex symmetric matrices whose imaginary part is positive definite, and let $\operatorname{Sp}(2 n, \mathbb{Z})$ denote the corresponding symplectic group with the action given by

$$
\left(\begin{array}{cc}
A & B \\
C & D
\end{array}\right) * Z=(A Z+B)(C Z+D)^{-1} .
$$

Received by the editors May 11, 1997.

2000 Mathematics Subject Classification. Primary 14K22; Secondary 32G13.

Both authors were supported in part by FONDECYT Grant \# 8970007 and Presidential Chair 1997.

(C)1999 American Mathematical Society 
We let $\mathcal{A}_{n}$ denote the quotient $\mathbb{H}_{n} / \operatorname{Sp}(2 n, \mathbb{Z})$; that is, the moduli space of principally polarized abelian varieties of dimension $n$. Then an element of $\mathcal{A}_{n}$ is (the class of) a pair $(A, H)$, where $A$ is a complex torus of dimension $n$ and $H$ is a nondegenerate principal Riemann form on $A$. In the sequel, we abbreviate principally polarized abelian variety by p.p.a.v.

An automorphism of $(A, H)$ is an automorphism of the complex torus $A$ which preserves the Riemann form $H$.

\section{RiEmann MATRICES}

Let $M_{n}$ denote the $\mathbb{Z}$-module $\bigoplus_{1}^{n} \mathbb{Z}$. Then the map $\phi: M_{n} \rightarrow M_{n+1}$ given by $\phi\left(x_{1}, x_{2}, \ldots, x_{n}\right)=\left(x_{1}, x_{2}, \ldots, x_{n},-\sum_{i=1}^{n} x_{i}\right)$ is $\mathbb{Z}$-linear and injective; its image is the hyperplane

$$
U=\left\{\left(y_{1}, y_{2}, \ldots, y_{n}, y_{n+1}\right) \in M_{n+1}: \sum_{i=1}^{n+1} y_{i}=0\right\} .
$$

By permutation of coordinates, each permutation in $S_{n+1}$ induces a $\mathbb{Z}$-linear map over $U$. If these maps are transported by $\phi$, then we obtain the standard representation (of degree $n$ ) of $S_{n+1}$, denoted $\widetilde{\theta}: S_{n+1} \rightarrow \mathrm{GL}_{\mathbb{Z}}\left(M_{n}\right)$.

The following result is clear.

Lemma 3.1. The direct sum of the representation $\widetilde{\theta}$ and its dual induces a faithful symplectic representation of the symmetric group

$$
\theta: S_{n+1} \longrightarrow \mathrm{Sp}(2 n, \mathbb{Z}) .
$$

In our next lemma we characterize the subvariety of $\mathbb{H}_{n}$, which is fixed pointwise by $\theta\left(S_{n+1}\right)$.

Lemma 3.2. Let $\theta$ be the given symplectic representation of $S_{n+1}$. Then the subvariety $\mathbb{H}_{n}(\theta)$ of $\mathbb{H}_{n}$ fixed pointwise under $\theta\left(S_{n+1}\right)$ is of dimension one and its elements are given by the Riemann matrices of the form

$$
Z_{\tau}=\tau\left(\begin{array}{rrrr}
n & -1 & \ldots & -1 \\
-1 & n & & \vdots \\
& & \ddots & -1 \\
-1 & \cdots & -1 & n
\end{array}\right)
$$

with $\Im \tau>0$.

Proof. For every permutation $g \in S_{n+1}$, let $m_{g}$ denote the corresponding $n \times n$ unitary integral matrix $\widetilde{\theta}(g)$. Then $\theta(g)=m_{g} \oplus^{t} m_{g}^{-1}$, where ${ }^{t}$ denotes the transpose of the given matrix.

Hence the action of $\theta(g)$ on $\mathbb{H}_{n}$ is given by $Z \longrightarrow m_{g} Z^{t} m_{g}$ and the result follows (for instance, by calculating with the generators $\alpha$ and $\gamma$ ).

Passing to the quotient $\mathcal{A}_{n}$, the pencil of Lemma 3.2 defines a one-dimensional family of p.p.a.v.'s and we obtain the following result.

Proposition 3.3. For each $n$ greater than or equal to two, there exists a pencil $\mathcal{A}_{n}(\theta)$ of p.p.a.v.'s of dimension $n$ such that, for each $A$ in $\mathcal{A}_{n}(\theta)$, $\operatorname{Aut}(A)$ contains $\theta\left(S_{n+1}\right)$.

Also, we can now prove the following two results. 
Corollary 3.4. For each $n$ greater than or equal to five, there are no Jacobians in $\mathcal{A}_{n}(\theta)$.

Proof. Let $C$ be a curve of genus $n$ at least five, and let $J(C)$ denote its Jacobian. If we assume that $J(C)$ belongs to $\mathcal{A}_{n}(\theta)$, then $\operatorname{Aut}(J(C))$ contains a subgroup $G$ isomorphic to $S_{n+1}$. But, by Torelli's theorem, Aut $(J(C))$ is either isomorphic to a $\mathbb{Z} / 2 \mathbb{Z}$-extension of $\operatorname{Aut}(C)$ or to $\operatorname{Aut}(C)$, which is a contradiction by the Hurwitz bound for $\operatorname{Aut}(C)$.

Corollary 3.5. For $n$ equal to four, the only curve whose Jacobian is contained in $\mathcal{A}_{4}(\theta)$ is Bring's curve.

Proof. We begin by recalling that Bring's curve, which we denote by $B$, is the unique curve of genus four such that its automorphism group contains (in fact, it is isomorphic to) a group isomorphic to $S_{5}$.

Following [9], a Riemann matrix for $J(B)$ may be taken as

$$
Z_{B}=\tau_{0}\left(\begin{array}{rrrr}
4 & 1 & -1 & 1 \\
1 & 4 & 1 & -1 \\
-1 & 1 & 4 & 1 \\
1 & -1 & 1 & 4
\end{array}\right)
$$

where $\tau_{0} \in \mathbb{H}_{1}$ is such that $j\left(\tau_{0}\right)=\frac{-25}{2}$ and that $j\left(5 \tau_{0}\right)=\frac{-5 \times 29^{3}}{2^{5}}$.

$$
\text { If } A=\left(\begin{array}{rrrr}
-1 & 1 & -1 & 1 \\
1 & 0 & 0 & 0 \\
0 & -1 & 0 & 0 \\
0 & 0 & 1 & 0
\end{array}\right) \text {, then, for } E=\left(\begin{array}{ll}
A & 0 \\
0 & { }^{t} A^{-1}
\end{array}\right) \text { in } \operatorname{Sp}(8, \mathbb{Z}) \text {, we }
$$

have that $E * Z_{B}=Z_{\tau_{0}}$ is in $\mathbb{H}_{4}(\theta)$.

Remark 3.6. The cases $n=3$ and $n=2$ are discussed later on. See Remark 4.4 and Section 6.

\section{Decomposition AND IRREducibiLity}

In this section we will study the different kinds of decompositions that the p.p.a.v.'s of $\mathcal{A}_{n}(\theta)$ admit.

Since there are different kinds of equivalences between Riemann matrices (or abelian varieties), and so as to avoid ambiguities, we begin by recalling the definitions involved in our results.

The two p.p.a.v.'s $\left(A_{1}, H_{1}\right)$ and $\left(A_{2}, H_{2}\right)$ are called isomorphic if there exists a surjective homomorphism $h: A_{1} \longrightarrow A_{2}$ with trivial kernel. They will be called isomorphic as principally polarized abelian varieties if there exists an isomorphism $h$ (as above) which further preserves the given polarizations $H_{1}$ and $H_{2}$.

A p.p.a.v. is called irreducible if it is not isomorphic as a principally polarized abelian variety to the product of p.p.a.v.'s of smaller dimensions.

We can now prove the following result.

Theorem 4.1. Each $A_{\tau}$ in $\mathcal{A}_{n}(\theta)$ that is induced by a Riemann matrix $Z_{\tau} \in \mathbb{H}_{n}(\theta)$ is isomorphic to the following product of $n$ elliptic curves:

$$
E_{(n+1) \tau} \times E_{(n+1) \tau} \times \cdots \times E_{(n+1) \tau} \times E_{\tau} .
$$


Proof. We consider the matrices $C, D$, and $M$ in $\operatorname{GL}(n, \mathbb{Z})$ as well as the $2 n \times 2 n$ unimodular matrix $N$ given by

$$
\begin{gathered}
C=\left(\begin{array}{ccccc}
2 & 1 & \ldots & 1 & 1 \\
1 & 2 & \ldots & 1 & 1 \\
& & \ldots & \\
1 & 1 & & 2 & 1 \\
1 & 1 & \ldots & 1 & 1
\end{array}\right), D=\left(\begin{array}{cccccc}
n+1 & 0 & \ldots & 0 & 0 \\
0 & n+1 & \ldots & 0 & 0 \\
& & \ldots & & \\
0 & 0 & & n+1 & 0 \\
0 & 0 & \ldots & 0 & 1
\end{array}\right), \\
M=\left(\begin{array}{ccccc}
1 & 0 & \ldots & 0 & 0 \\
0 & 1 & \ldots & 0 & 0 \\
0 & 0 & \ldots & 1 & 0 \\
1 & 1 & \ldots & 1 & 1
\end{array}\right) \text { and } N=\left(\begin{array}{cc}
C & 0 \\
0 & M
\end{array}\right), \text { respectively. }
\end{gathered}
$$

Then we have

$$
C\left(I_{n} Z_{\tau}\right)=\left(I_{n} \tau D\right) N
$$

for $Z_{\tau} \in \mathbb{H}_{n}(\theta)$, and the result follows.

The problem of finding Jacobians which are isomorphic (and not only isogenous) to the product of elliptic curves, for any value of the genus of the corresponding curve, is a difficult one (cf. [4]).

As far as the authors have been able to learn from the literature, it was known to be possible up to genus three (cf. [2]).

Our next result gives an example for genus four.

Corollary 4.2. Let B be the Bring's curve, as in Corollary 3.5. Then the Jacobian of $B$ is isomorphic to the product of elliptic curves.

It is well known that each p.p.a.v. $(A, H)$ of dimension $n$ is isomorphic as a principally polarized abelian variety to a unique product of irreducible p.p.a.v.'s $\prod_{i=1}^{s}\left(A_{i}, H_{i}\right)$ (cf. [1]); this is the decomposition of the theta divisor associated to the polarization $H$ of $A$.

We now prove that, for $n$ at least three, each $A \in \mathbb{H}_{n}(\theta)$ is irreducible as a principally polarized abelian variety. Remark that the irreducibility is well known for the Jacobian of a curve [5]. Notwithstanding, we already know that: our pencil does not contain Jacobians for $n \geq 5$, and, for $n=4$, it only contains one; now, for $2 \leq n \leq 3$, we only know a priori that the generic points of $\mathbb{H}_{2}(\theta)$ and $\mathbb{H}_{3}(\theta)$ are Jacobians.

Theorem 4.3. For each $n$ greater than or equal to three, every $A$ in $\mathcal{A}_{n}(\theta)$ is irreducible as a principally polarized abelian variety.

Proof. Let $(A, H)$ be in $\mathcal{A}_{n}(\theta)$ and assume that $(A, H)$ is reducible as a principally polarized abelian variety. Then it is isomorphic to a product $\left(A_{1}, H_{1}\right) \times\left(A_{2}, H_{2}\right) \times$ $\cdots \times\left(A_{s}, H_{s}\right)$, where each $\left(A_{i}, H_{i}\right)$ is an irreducible p.p.a.v. of dimension $n_{i}$, with $n_{i}<n$ and $n_{1}+n_{2}+\cdots+n_{s}=n$.

Since the group $S_{n+1}$ acts on the tangent space at the origin of $A$ and since the standard representation is irreducible, the action must permute transitively the set of components $\left\{\left(A_{1}, H_{1}\right), \ldots,\left(A_{s}, H_{s}\right)\right\}$.

If we call $F$ the isotropy subgroup of one component $\left(A_{k}, H_{k}\right)$, then $F$ is a proper subgroup of index $\gamma$ in $S_{n+1}$, with $2 \leq \gamma \leq n$, and the action of $S_{n+1}$ on the tangent space is induced by $F$. 
We first assume that $n \geq 4$. Then $F$ is isomorphic to the alternating group $A_{n+1}$, hence we obtain a contradiction.

For $n$ equal to three, the group $F$ may be isomorphic to $A_{4}$ or to $D_{4}$ (the dihedral group of order 8 ) and, again, these subgroups cannot induce the given representation.

Remark 4.4. While writing this proof, the authors learned that it had been shown (cf. [8]) that the p.p.a.v.'s of dimension three with standard action of $S_{4}$ are all Jacobians, which gives another proof of the irreducibility in the particular case $n=3$.

If we consider $n$ equal to two, then the standard representation of $S_{3}$ is induced by the cyclic subgroup of order three and $A$ could be isomorphic as a principally polarized abelian variety to the product $E_{\omega} \times E_{\omega}$, where $E_{\omega}$ is the elliptic curve such that $j(\omega)=0$. We will see that this case occurs for special values of $\tau$, but, in order to give the result in a precise form, we will postpone the analysis until Section 6 .

\section{Uniformization FOR $A_{n}(\theta)$}

In this section we will discuss a geometric description of the pencil $A_{n}(\theta)$ of p.p.a.v.'s that admit the standard action of $S_{n+1}$.

Let $N_{\mathrm{Sp}}(\theta)$ denote the normalizer of the subgroup $\theta\left(S_{n+1}\right)$ in $\operatorname{Sp}(2 n, \mathbb{Z})$, and let $\operatorname{Aut}_{\mathrm{Sp}}(\theta)$ denote the group of automorphisms of $S_{n+1}$ that are realized inside $\operatorname{Sp}(2 n, \mathbb{Z})$. Note that all automorphisms of $\operatorname{Sp}(2 n, \mathbb{Z})$ are inner (cf. [7]).

We begin by describing the centralizer of $\theta\left(S_{n+1}\right)$.

For each $M \in N_{\mathrm{Sp}}(\theta)$ we may define an automorphism $f_{M}: \theta\left(S_{n+1}\right) \rightarrow \theta\left(S_{n+1}\right)$ by $f_{M}(X)=M X M^{-1}$. Hence we have the following exact sequence of groups:

$$
\{e\} \rightarrow \operatorname{ker} \psi \rightarrow N_{\mathrm{Sp}}(\theta) \stackrel{\psi}{\rightarrow} \operatorname{Aut}_{\mathrm{Sp}}(\theta) \rightarrow\{e\},
$$

with $\psi(M)=f_{M}$ for each $M$ in $N_{\mathrm{Sp}}(\theta)$ and where the map $\operatorname{ker} \psi \rightarrow N_{\mathrm{Sp}}(\theta)$ is the natural inclusion.

We can now describe the kernel of $\psi$ (or, equivalently, the centralizer of $\theta\left(S_{n+1}\right)$ in $\operatorname{Sp}(2 n, \mathbb{Z}))$.

Lemma 5.1. The subgroup $\operatorname{ker} \psi$ is isomorphic to the modular group $\Gamma_{0}(n+1)$.

Proof. We consider $M=\left(\begin{array}{cc}A & B \\ C & D\end{array}\right)$ in $\operatorname{Sp}(2 n, \mathbb{Z})$. If $M$ is in ker $\psi$, then

$$
M \theta(g) M^{-1}=\theta(g) \text {, for every } g \in S_{n+1} \text {. }
$$

Recalling the notation used in the proof of Lemma 3.2, $\theta(g)=m_{g} \oplus{ }^{t} m_{g}^{-1}$, we obtain that (5.1) is equivalent to the equalities $A m_{g}=m_{g} A, B{ }^{t} m_{g}^{-1}=m_{g} B$, $C m_{g}={ }^{t} m_{g}^{-1} C$ and $D{ }^{t} m_{g}^{-1}={ }^{t} m_{g}^{-1} D$, for every $g \in S_{n+1}$.

Since the standard representation $\widetilde{\theta}(g)=m_{g}$ given in Section 3 is irreducible, we obtain that $A=p I_{n}$, with $p \in \mathbb{Z}$, and that $D=s I_{n}$, with $s \in \mathbb{Z}$, where $I_{n}$ is the $n \times n$ identity matrix. 
An elementary calculation shows that $B=q U_{n}$ and $C=r V_{n}$, where $q$ and $r$ are integers and

$$
U_{n}=\left(\begin{array}{rrrr}
n & -1 & \ldots & -1 \\
-1 & n & \ldots & \vdots \\
\vdots & & \ddots & -1 \\
-1 & \ldots & -1 & n
\end{array}\right) \quad, \quad V_{n}=\left(\begin{array}{cccc}
2 & 1 & \ldots & 1 \\
1 & 2 & \ldots & \vdots \\
\vdots & & \ddots & 1 \\
1 & \ldots & 1 & 2
\end{array}\right)
$$

Note that $U_{n} V_{n}=(n+1) I_{n}$.

Since $M$ is symplectic, we obtain that $p s I_{n}-r q(n+1) I_{n}=I_{n}$; therefore we may define the isomorphism $h: \Gamma_{0}(n+1) \rightarrow \operatorname{ker} \psi$ by $h\left(\begin{array}{cc}p & q \\ r(n+1) & s\end{array}\right)=$

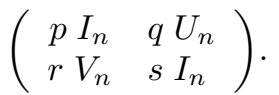

Since the group $S_{n+1}$ is complete for $n \neq 1$ and $n \neq 5$, we can identify $\operatorname{Aut}_{\mathrm{Sp}}(\theta)$ with $\theta\left(S_{n+1}\right)$. Furthermore $\theta\left(S_{n+1}\right)$ is centerless, therefore $\operatorname{ker} \psi \cap \theta\left(S_{n+1}\right)=\{e\}$. For $n=1$, we have $\operatorname{Aut}\left(S_{2}\right)=1$, and for $n=5$, a calculation shows that no external automorphism of $S_{6}$ is realized in $\operatorname{Sp}(10, \mathbb{Z})$. Hence we have obtained our next result.

Theorem 5.2. For $n$ greater than or equal to two, $N_{\mathrm{Sp}}(\theta)$ is isomorphic to the semidirect product $\Gamma_{0}(n+1) \ltimes \theta\left(S_{n+1}\right)$.

Remark 5.3. The natural morphism $\mathbb{H}_{n}(\theta) \longrightarrow \mathbb{H}_{1}$ which sends $Z_{\tau}$ to $\tau$ is equivariant under the action of ker $\psi$ on $\mathbb{H}_{n}(\theta)$, hence there is an induced injective morphism

$$
\mathbb{H}_{n}(\theta) / \operatorname{ker} \psi=A_{n}(\theta) \longrightarrow \mathbb{H}_{1} / \Gamma_{0}(n+1)=X_{0}(n+1) .
$$

We now use a beautiful geometric idea suggested to us by A. Beauville to show that this last morphism is also surjective.

Theorem 5.4. Let $n$ be greater than or equal to two. Then $A_{n}(\theta)$ is isomorphic to $X_{0}(n+1)$.

Proof. Let $(E, G)$ be a point of the modular curve $X_{0}(n+1)$. Then $E$ is an elliptic curve and $G$ is a cyclic subgroup of order $(n+1)$ of $E$.

Let $E^{n+1}$ be the abelian variety given by the product of $n+1$ copies of $E$ with the principal (product) polarization $P$. In $E^{n+1}$ we consider the abelian subvariety $A$ given by

$$
A=\left\{\left(x_{1}, x_{2}, \ldots, x_{n}, x_{n+1}\right) \in E^{n+1}: \sum_{i=1}^{n+1} x_{i}=0\right\},
$$

with the polarization $P_{A}$ induced by the polarization $P$ of $E^{n+1}$. Note that $S_{n+1}$ acts on $E^{n+1}$ by permutation of coordinates and that this action restricts to $A$.

If $\widehat{A}$ denotes the dual abelian variety of $A$, then $P_{A}$ induces an isogeny $P_{A}$ : $A \longrightarrow \widehat{A}$ with kernel given by the points of order $n+1$ of $E$ embedded in the diagonal of $A$. We denote this embedded group by $N$.

Furthermore, the image of the subgroup $G$ in $N$ (which we again denote by $G$ ) is totally isotropic for the Weil form. Then (cf. [6]) there exists a principal polarization $Q$ on $A / G$ whose inverse image is $P_{A}$. 
It can also be verified (by a change of coordinates) that $A / G$ is isomorphic to the product $E^{n-1} \times E / G$.

Since the construction is equivariant under the action of $S_{n+1}$, we have that $(A / G, Q)$ belongs to $A_{n}(\theta)$, and the result follows.

\section{THE CASE $n=2$}

We now study the irreducibility of the members of $A_{n}(\theta)$ for the interesting special case $n=2$.

Discussions with R. Salvati Manni were especially helpful to understand this case.

Proposition 6.1. For $n$ equal to two, each p.p.a.v. $A_{\tau}$ in $A_{2}(\theta)$ is irreducible as a p.p.a.v., for $\tau$ non-equivalent to $\tau_{0}=\frac{-3+i \sqrt{3}}{6} \bmod \Gamma_{0}(3)$.

If $\tau$ is equivalent to $\tau_{0} \bmod \Gamma_{0}(3)$, then $A_{\tau}$ is isomorphic as a principally polarized abelian variety to $E_{\omega} \times E_{\omega}$, where $E_{\omega}$ is the elliptic curve with $j(\omega)=0$.

Proof. Let $A_{\tau}$ be in $A_{2}(\theta)$ and represented by the Riemann matrix $Z_{\tau} \in \mathbb{H}_{2}(\theta)$.

If we assume that $A_{\tau}$ is reducible, then it must be isomorphic as a principally polarized abelian variety to $E_{\omega} \times E_{\omega}$, where $E_{\omega}$ is an elliptic curve with $j(\omega)=0$. Let $m \in \operatorname{Sp}(4, \mathbb{Z})$ be a matrix inducing this isomorphism.

Now the p.p.a.v. $E_{\omega} \times E_{\omega}$ admits the automorphisms $S=\left(\begin{array}{cccc}0 & 1 & 0 & 0 \\ 1 & 0 & 0 & 0 \\ 0 & 0 & 0 & 1 \\ 0 & 0 & 1 & 0\end{array}\right)$, $T=\left(\begin{array}{rrrr}1 & 0 & 0 & 0 \\ 0 & -1 & 0 & 0 \\ 0 & 0 & 1 & 0 \\ 0 & 0 & 0 & -1\end{array}\right)$, and $R=\left(\begin{array}{rrrr}0 & 0 & 1 & 0 \\ 0 & 0 & 0 & 1 \\ -1 & 0 & -1 & 0 \\ 0 & -1 & 0 & -1\end{array}\right)$.

Furthermore, these automorphisms satisfy the relations

$$
T^{2}=I_{4}, T R=R T \text {, and } T S=-S T .
$$

But then the automorphism group of $A_{\tau}$ must contain $n=m T m^{-1}$ and, by construction, $\theta\left(S_{3}\right)$. Of course, the relations (6.1) must be satisfied by the corresponding elements of $\operatorname{Aut}\left(A_{\tau}\right)$.

These relations, together with the fact that $n$ belongs to $\Gamma_{2}(2,4)$, allow us to conclude that $n$ must have the following form:

$$
n=\left(\begin{array}{rrrr}
a & 2 a & 0 & 2 b \\
-2 a & -a & -2 b & 0 \\
0 & -2 c & a & -2 a \\
2 c & 0 & 2 a & -a
\end{array}\right)
$$

with $a, b, c$ integers and satisfying $4 b c-3 a^{2}=1$.

Since $n$ fixes $Z_{\tau}$, a calculation shows that $\tau$ is equivalent to $\tau_{0}=\frac{-3+i \sqrt{3}}{6} \bmod$ $\Gamma_{0}(3)$. 
For an explicit example, we take

$$
n=\left(\begin{array}{rrrr}
1 & 2 & 0 & 2 \\
-2 & -1 & -2 & 0 \\
0 & -2 & 1 & -2 \\
2 & 0 & 2 & -1
\end{array}\right) \quad \text { and } m=\left(\begin{array}{rrrr}
1 & 0 & -1 & 1 \\
0 & -1 & 1 & -1 \\
1 & 0 & 0 & -1 \\
0 & 1 & -1 & 0
\end{array}\right)
$$

\section{REFERENCES}

1. C. H. Clemens and P. A. Griffiths, The intermediate Jacobian of the cubic threefold, Annals of Mathematics 95 (1972), 281-356. MR 46:1796

2. C. J. Earle, H. E. Rauch, Function Theorist, Differential Geometry and Complex Analysis, Chavel, edited by I. Kra and H. M. Farkas, Springer-Verlag, 1985, pp. 15-31. MR 86h:01063

3. C. J. Earle, Some Jacobians which split, Lecture Notes in Mathematics 747, Springer-Verlag, 101-107. MR 80m:14018

4. T. Ekedahl and J.-P. Serre, Exemples de courbes algébriques à jacobienne complètement décomposable, C. R. Acad. Sci. Paris t. 137 Série I (1993), 509-513. MR 94j:14029

5. H. H. Martens, Torelli's Theorem and a generalization for hyperelliptic surfaces, Comm. Pure Appl. Math. 16 (1963), 97-110. MR 27:2623

6. L. Moret-Bailly, Famille de courbes et des variétés abeliennes sur $\mathbb{P}_{1}$, Asterisque 86 (1981), 109-124.

7. I. Reiner, Automorphisms of the symplectic modular group, Transactions of the A.M.S. 80 (1955), 35-50. MR 17:458a

8. J. F. X. Ries, The splitting of some Jacobi varieties using their automorphism group, Contemp. Math. 201 (1997), 81-124. MR 98c:14022

9. G. Riera and R. E. Rodríguez, The period matrix of Bring's curve, Pacific J. of Math. 154 (1992), 179-200. MR 93e:14033

10. R. E. Rodríguez and V. González-Aguilera, Fermat's quartic curve, Klein's curve and the Tetrahedron, Contemp. Math. 201 (1997), 43-62. MR 97j:14033

Departamento de Matemáticas, Universidad Técnica Federico Santa María, Casilla 110-V, Valparaíso, Chile

E-mail address: vgonzale@mat.utfsm.cl

Facultad de Matemáticas, Pontificia Universidad Católica de Chile, Casilla 306, Correo 22, Santiago, Chile

E-mail address: rubi@mat.puc.cl 